\title{
Critique et mémoire : les romanciers et l'histoire du roman
}

Criticism and memory: novelists and the history of fiction

\section{Isabelle Daunais}

\section{(2) OpenEdition}

\section{Journals}

Édition électronique

URL : http://journals.openedition.org/tangence/576

ISSN : 1710-0305

\section{Éditeur :}

Université du Québec à Rimouski, Université du Québec à Trois-Rivières

Édition imprimée

Date de publication : 1 décembre 2018

Pagination : 13-25

ISSN : 1189-4563

Référence électronique

Isabelle Daunais, «Critique et mémoire : les romanciers et l'histoire du roman », Tangence [En ligne], 118 | 2018, mis en ligne le 01 décembre 2019, consulté le 03 décembre 2019. URL : http:// journals.openedition.org/tangence/576

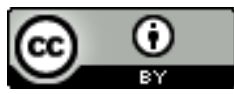

La revue Tangence est mise à disposition selon les termes de la Licence Creative Commons Attribution 4.0 International. 


\section{Critique et mémoire: les romanciers et l'histoire du roman \\ Isabelle Daunais \\ Université McGill}

De nombreux travaux ont été publiés, au cours des dernières années, sur ce qu'on peut appeler, avec Michel Murat, Vincent Debaene, Jean-Louis Jeannelle et Marielle Macé, l'histoire «indigène» de la littérature ${ }^{1}$, c'est-à-dire l'histoire de la littérature telle que proposée par les écrivains eux-mêmes. On pense ici au numéro de la Revue des sciences humaines consacré aux "Écrivains critiques» ou à ceux de La Licorne sur les "Fictions d'histoire littéraire» et de la Romanic Review sur les "Literary Histories of Literature ${ }^{2}$. Ces travaux s'intéressent aux rapports que les écrivains, en tant que personnes de métier, entretiennent avec l'acte critique, et à ce qui distingue leur vision de l'histoire de la littérature de celle proposée par la critique savante. S'ils portent aussi bien sur la manière dont les créateurs pensent l'histoire littéraire que sur les formes par lesquelles et au sein desquelles s'exprime cette réflexion, la question des genres y est rarement abordée. Les genres, pourtant, n'ont pas tous la même histoire, ni ne se développent suivant les mêmes contraintes, les mêmes élans ou les mêmes découvertes esthétiques.

1. Vincent Debaene, Jean-Louis Jeannelle, Marielle Macé et Michel Murat (dir.), L'histoire littéraire des écrivains, Paris, Presses universitaires Paris-Sorbonne, 2013.

2. Marie-Paule Berranger (dir.), Revue des sciences humaines, $\mathrm{n}^{\circ} 306$ (L'écrivain critique), 2012; Jean-Louis Jeannelle (dir.), La Licorne, no 86 (Fictions d'histoire littéraire), 2009; Antoine Compagnon et Vincent Debaene (dir.), Romanic Review, vol. 100, $\mathrm{n}^{\text {os }} 1-2$ (Literary Histories of Literature), 2010. 
Le roman, à cet égard, suit une évolution historique qu'on pourrait décrire comme étant à contretemps: il acquiert son plein prestige de façon tardive, au XIX ${ }^{\mathrm{e}}$ siècle, pour le voir remettre en question, sous la forme du "soupçon» - selon le terme bien connu de Nathalie Sarraute - dès le siècle suivant. Les réflexions qui suivent visent à proposer quelques hypothèses sur le rapport à l'histoire, et plus spécifiquement à la mémoire et à l'héritage, qu'induit cette situation et ce, au moment où elle trouve, au tournant $\mathrm{xx}^{\mathrm{e}}$ du siècle, une forme de cristallisation.

Pour ce faire, j'aimerais partir d'un article de Judith Schlanger, intitulé "Devant le temps des écrivains», qui interroge les raisons pour lesquelles, «au même moment et dans le même espace culturel, certains écrivains s'intéressent à la littérature dans son histoire et en parlent - et d'autres pas $^{3}{ }^{\prime}$, autrement dit, pourquoi l'écriture de leur œuvre est, pour certains écrivains, indissociable d'une mémoire des lettres qu'il leur importe de saisir et d'évoquer, alors que pour d'autres, aussi admiratifs puissent-ils être des œuvres du passé, le «corpus littéraire n'est pas une présence.» (DTE, p. 29)

Dans cette réflexion que propose Judith Schlanger sur l'intérêt que les écrivains portent ou non à ce qu'on peut appeler l'héritage littéraire, deux éléments frappent d'emblée. Le premier est que tous les écrivains qu'elle convoque sont des romanciers, la seule exception étant Charles Péguy, qu'elle cite pour son idée de ministère du présent, soit «le pouvoir propre au présent de faire surgir du réel ici et maintenant» (DTE, p. 34) par lequel le poète invitait ses contemporains à lire les œuvres du passé - et surtout celles des classiques non pas comme des œuvres héritées, mais comme des œuvres du présent et par là à les lire sans la médiation des apparats critiques, des éditions savantes ou du discours historique. Schlanger ne vise pourtant en rien dans sa réflexion la spécificité du roman et n'aborde jamais la question des genres; mais il reste que, par les exemples fournis, c'est «le temps des romanciers» plutôt que celui des écrivains en général qu'elle se trouve à interroger. Le second élément est que les romanciers qui entrent dans la catégorie des «non-penseurs» de l'histoire de leur art ne sont ni hostiles au passé ni spécifiquement

3. Judith Schlanger, «Devant le temps des écrivains», The Romanic Review, vol. $100, \mathrm{n}^{\mathrm{os}} 1-2,2010, \mathrm{p} .29$. Désormais, les références à cet article seront indiquées par le sigle DTE, suivi de la page, et placées entre parenthèses dans le corps du texte. 
défenseurs d'une conception actuelle de l'art et de la littérature, mais penseurs et défenseurs d'une valeur située sur une autre axiologie, celle de la spontanéité, par laquelle ils revendiquent leur exception. Ce sont, écrit Schlanger, des écrivains "qui se posent comme une pure nature: un tempérament, une verve, un timbre, un ton, une voix. Littérature d'épanchement, de confession, d'expression de soi, d'autobiographie.» (DTE, p. 29) Ces «artistes confessionnels» (DTE, p. 29), ainsi qu'elle les nomme, peuvent avoir beaucoup lu, et même se reconnaître quelques dettes à l'endroit de tel ou tel auteur dont ils admettent l'influence ou l'inspiration, mais ils ne s'intéressent ni à l'histoire de leur art ni à l'héritage qu'il constitue. Entrent aussi dans cette catégorie les écrivains qu'elle appelle «ingénus", c'est-à-dire ceux que rien, qu'il s'agisse de leur éducation, de leur milieu ou de leur parcours, ne prépare à une carrière littéraire, mais qui tirent profit pour témoigner de leur vie d'un talent natif en même temps que d'une concordance affective avec leur époque, comme en offrent les exemples d'une Albertine Sarrazin ou d'une Marguerite Audoux, ouvrière dans une filature et lauréate en 1910 du prix Femina pour un récit intitulé Marie-Claire, inspiré de son enfance d'orpheline et de bergère.

Judith Schlanger ne propose pas d'appellations spécifiques ou de sous-catégories pour les romanciers penseurs de l'histoire de leur art. Sans doute l'ensemble qu'ils composent est-il trop vaste et les formes de leurs réflexions trop multiples pour qu'une telle opération soit possible, mais cette absence tient surtout à ce que son propos est de suggérer qu'il est plus attendu, plus normal pour un écrivain (en l'occurrence pour un romancier), non seulement d'écrire dans la pensée de l'histoire de son art, mais aussi de faire de cette pensée l'objet d'une réflexion ouverte et partagée. L'idée d'une telle norme paraît à première vue étonnante, les cas de "non-pensée» pesant beaucoup plus, numériquement du moins, dans la production courante, que les cas de "pensée »: à côté des romanciers qui expriment et revendiquent une réflexion sur leur art (sous forme d'essais, d'articles, préfaces, entretiens), se trouvent un nombre beaucoup plus grand de romanciers silencieux. On pourrait même arguer que les «artistes confessionnels» et «ingénus », loin de constituer des exceptions, sont plutôt ceux qui, aujourd'hui, constituent la norme romanesque, tant l'ingénuité (ou si l'on préfère l'authenticité) et la confession se sont imposées, dans le roman $\mathrm{du}_{\mathrm{xx}} \mathrm{x}^{\mathrm{e}}$ siècle, 
comme des valeurs fortes. Mais outre que la question du nombre n'est pas seule en jeu (les artistes confessionnels, qui ont pour «sujet perpétuel et unique [...] leur expérience personnelle et leur réaction à tout» [DTE, p. 29], peuvent multiplier leurs récits à l'infini sans apporter quoi que ce soit de neuf), il reste que, en dehors de toute proportion ou de toute statistique, la pensée des romanciers sur leur art, lorsqu'elle se manifeste, a très souvent pour objet l'histoire du roman, ses filiations et ses legs, en un mot sa mémoire.

En fait, de Gide défenseur de Dostö̈evski à Kundera admirateur de Cervantès et de Diderot, en passant par Henry James pour qui Flaubert est le prince des romanciers ( the novelist's novelist ${ }^{4} »$ ), Proust lecteur de Balzac, Gracq de Stendhal ou Sartre de Faulkner, la liste des romanciers pour qui réfléchir à l'art du roman est réfléchir à un héritage est si longue qu'il serait possible de formuler pour le roman une hypothèse inverse de celle avancée naguère par Harold Bloom à propos de la poésie. Si les poètes, comme le suggère Bloom, craignent d'être «influencés » par leurs devanciers ${ }^{5}$, c'est-à-dire sont mus par l'idéal de créer une œuvre parfaitement neuve, abstraite de toute lignée dont elle serait l'héritière et le produit, les romanciers, selon cette hypothèse contraire, non seulement n'hésiteraient pas à se reconnaître des maîtres et des prédécesseurs, mais redouteraient de se retrouver sans lien avec eux, de perdre la mémoire de leur art et le sens de sa continuité. Pour le dire autrement, si les romanciers sont «inquiets» ou «anxieux» de quelque chose, ce ne serait pas de ne pas se sentir neufs, de ne pas débarquer sur la scène littéraire avec des œuvres inédites, ce serait d'oublier tout ce que le roman a inventé comme formes et opéré comme découvertes au fil de son histoire, ce serait de rompre le lien qui les rattache au passé.

À quoi tient cet attachement des romanciers, ou en tout cas d'un grand nombre d'entre eux, à la mémoire de leur art? D'où vient qu'il traverse et souvent motive le vaste ensemble des commentaires qu'ils consacrent au roman, du XIx ${ }^{e}$ siècle jusqu'à nous? Et en quoi peuton le considérer comme singulier? Car cette mémoire ne doit pas être confondue avec celle qui puiserait à une source historique qu'il s'agirait de maintenir ou de faire revivre (à l'instar, par exemple,

4. Henry James, «Gustave Flaubert», dans Madame Bovary, trad. de l'anglais par William Blaydes, New York, Collier, 1902, p. 402.

5. Harold Bloom, The Anxiety of Influence. A Theory of Poetry, Londres, Oxford University Press, 1973. 
de celle des auteurs de la Renaissance pour l'Antiquité). Lorsque Stendhal et Flaubert évoquent Don Quichotte, ils ne cherchent pas à retrouver une forme perdue, encore moins à l'imiter, mais à voir comment, dans certaines de ses incarnations du moins, elle reste vivante (Stendhal rappelle, dans la Vie de Henry Brulard, combien la lecture de Cervantès, au temps de sa jeunesse, le mettait en joie: «Qu'on juge de l'effet de Don Quichotte au milieu d'une si horrible tristesse! La découverte de ce livre, lu sous le second tilleul de l'allée du côté du parterre, dont le terrain s'enfonçait d'un pied, et là [où] je m'asseyais, est peut-être la plus grande époque de ma vie ${ }^{6}$.» Flaubert fait preuve d'un enthousiasme similaire: «Ce qu'il y a de prodigieux dans Don Quichotte, c'est l'absence d'art et cette perpétuelle fusion de l'illusion et de la réalité qui en fait un livre si comique et si poétique $^{7}$ », écrit-il à Louise Colet, en 1852). En réponse à ces questions, j'aimerais proposer que cette pensée critique mémorielle du roman survient dans la foulée de la "réussite» du roman comme genre majeur - réussite qui, en plus de faire du romancier une figure de plus en plus notable de la scène littéraire et par là appelée à intervenir elle-même sur le rôle de son art, entraîne le besoin d'ordonner une production toujours croissante (par des comptes rendus, des appréciations, des analyses, mais aussi une histoire), de la canaliser, d'en montrer la direction, de suggérer des filiations. Elle est aussi alimentée par la «crise ${ }^{8} »$, suivant l'expression désormais célèbre de Michel Raimond, que connaît le roman à la fin du XIX ${ }^{e}$ siècle. Confrontés à l'épuisement de la veine naturaliste du siècle précédent et pressés de se prononcer sur l'avenir du roman par une institution littéraire (critiques, journaux) de plus en plus sensible aux mouvements d'avantgarde, les romanciers sont amenés à réfléchir aux spécificités de leur pratique, à ses possibilités, à son évolution. Cette réflexion est également favorisée par le fait que l'héritage littéraire (le patrimoine des grandes œuvres censées être connues de tous ceux qui disent appartenir au public lettré) commence, au même moment, à s'encombrer de telle façon qu'il n'est plus possible pour quiconque de posséder

6. Stendhal, Vie de Henry Brulard, dans Euvres intimes, éd. Victor del Litto, Paris, Gallimard, coll. «Bibliothèque de la Pléiade», 1981, p. 618.

7. Lettre à Louise Colet, 22 novembre 1852, Correspondance, t. II, Paris, Gallimard, coll. «Bibliothèque de la Pléiade», 1980, p. 179.

8. Michel Raimond, La crise du roman. Des lendemains du naturalisme aux années vingt, Paris, Corti, 1966. 
directement et surtout totalement les œuvres du passé, même du passé proche. L'usage classique des lettres, grâce auquel les œuvres du passé étaient pour l'écrivain et le lecteur aussi vivantes que celles du présent, vit au cours du XIX ${ }^{\mathrm{e}}$ siècle ses dernières heures (illustrées dans $\grave{A}$ la recherche $d u$ temps perdu par la compagnie familière mais également excentrique - au point, justement, d'être relevé comme un fait saillant - que constituent pour la grand-mère du narrateur les lettres de Mme de Sévigné, auxquelles elle «était venue [...] par le dedans, par l'amour pour les siens, pour la nature ${ }^{9}$ » et qu'elle cite comme si elles lui étaient aussi proches que si elles lui étaient contemporaines). Sans doute est-ce quelques décennies plus tard, à partir des années 1940 et 1950, que le rapport classique au patrimoine lettré cédera tout à fait la place à de nouvelles façons d'entretenir la mémoire des lettres, ainsi qu'en témoigne la pratique qui se met alors à fleurir de réunir en listes, collections, "encyclopédies» ou «bibliothèques» (que ce soit celle, méthodique et savante, de la Pléiade ou celle, «idéale», de Raymond Queneau) ce qu'on estimera être la part la plus précieuse ou la plus représentative de l'héritage littéraire. Mais outre que l'idée d'encombrement apparaît dès la fin du $\mathrm{XIX}^{\mathrm{e}}$ siècle (par exemple chez Nietzsche pour qui «nulle génération ne vit encore un pareil spectacle, spectacle impossible à dominer du regard, comme celui que montre aujourd'hui la science du devenir universel. [...] L'homme moderne, en fin de compte, traîne avec lui une énorme masse de cailloux, les cailloux de l'indigeste savoir qui, à l'occasion, font entendre un bruit sourd, comme il est dit dans la fable $\left.{ }^{10} »\right)$, les signes concrets de cette transformation surviennent très tôt au début du $\mathrm{xx}^{\mathrm{e}}$ siècle, avec par exemple le "jeu de l'île déserte», qui invite chacun à nommer les quelques ouvrages qu'il emporterait avec lui s'il s'y trouvait contraint et dont Gide aurait lancé la mode en proposant, dans un article de La Nouvelle Revue française paru en $1913^{11}$, la liste qu'il dresserait lui-même advenant qu'il lui faille choisir les seuls dix romans qu'il pourrait emporter sans retour

9. Marcel Proust, À l'ombre des jeunes filles en fleurs, Paris, Gallimard, coll. «Folio », 1988 , p. 222.

10. Friedrich Nietzsche, Seconde considération intempestive. De l'utilité et de l'inconvénient des études historiques pour la vie [1874], Paris, Garnier-Flammarion, 1988, p. 103-104.

11. André Gide, "Les dix romans français que...», La Nouvelle Revue française [ $1^{\mathrm{er}}$ avril 1913], dans Essais critiques, éd. Pierre Masson, Paris, Gallimard, coll. «Bibliothèque de la Pléiade», 1999, p. 268-273. 
avec lui. Dans un monde et dans un temps où la mémoire des lettres demande, par sa lourdeur croissante, à être allégée, il n'est donc pas étonnant - il est même logique — de voir les écrivains devenir aussi des critiques, c'est-à-dire trier parmi les ouvres, y tracer des chemins, agir, à l'instar des professeurs dont le magistère se met en place au même moment ${ }^{12}$, en juges et gardiens de l'histoire littéraire. Car non seulement pèse sur cette histoire la menace de l'oubli, mais, dès lors qu'on ne peut pas tout conserver et qu'il faut choisir, dès lors qu'on ne peut emporter sur l'île trop peuplée du présent que dix, cinquante ou cent livres, se pose aussi la question de la concurrence. Défendre Dostoïevski face aux œuvres de Paul Bourget (Gide), Balzac contre Sainte-Beuve (Proust), le roman d'aventure plutôt que le roman symboliste (Jacques Rivière), ce n'est pas seulement prendre un parti esthétique plutôt qu'un autre, c'est aussi chercher à tenir le fil de ce que l'on considère essentiel de conserver pour la suite de la littérature et la continuité du roman.

Mais cette volonté de maintenir une direction et une mémoire en dépit d'un nécessaire désencombrement n'est logique qu'en apparence, puisqu'au même moment d'autres genres et d'autres arts fondent à l'inverse leur avenir sur la chance offerte par la nécessité où ils se trouvent de s'alléger et notamment s'alléger du passé. Paul Valéry, par exemple, se réjouit d'avoir vu «enfin, vers le milieu du $\mathrm{XIX}^{\mathrm{e}}$ siècle, se prononcer dans notre littérature une volonté remarquable d'isoler définitivement la Poésie de toute autre essence qu'ellemême " et qu'à travers elle on ait «véritablement déchiffré la signification d'ensemble des labeurs de nos ancêtres. ${ }^{13}$ » Pourquoi, lorsqu'il s'agit de comprendre le labeur des leurs, les romanciers - qu'il s'agisse de James, Stevenson, Gide, Proust, Virginia Woolf —, loin de chercher à isoler quoi que ce soit, loin de penser à des «essences» ou de vouloir trouver une "signification d'ensemble» qui résume et contienne (au double sens du terme) tout le passé, cherchent-ils plutôt à comprendre de quelle façon concrète leurs devanciers ont écrit des romans et de quelles «leçons» (pour emprunter le terme

12. Voir Antoine Compagnon, La Troisième République des lettres. De Flaubert à Proust, Paris, Seuil, 1983.

13. Paul Valéry, «Avant-propos à la Connaissance de la déesse» [1920], dans Variété. Euvres, éd. Jean Hytier, Paris, Gallimard, coll. "Bibliothèque de la Pléiade», 1957, t. I, p. 1271 et 1275. 
qu'utilise Henry James à propos de l'œuvre de Balzac ${ }^{14}$ ) précises ils peuvent s'inspirer?

Cette différence d'attitude face au legs du passé peut s'expliquer par le fait que la mémoire du roman est matériellement plus menacée que celle des autres arts. Si varié soit-il, le corpus poétique n'est pas aussi «informe» que le corpus romanesque, qu'aucune tradition n’ordonne ni ne hiérarchise. La poésie peut au demeurant exister en dehors des poèmes eux-mêmes, à travers ses principes ou sa technique (prosodie, rythme, rime, versification), là où le roman n'existe qu'à travers ses œuvres. Pour «se souvenir» de ce que peut être un roman, pour se rappeler ses déclinaisons possibles (et à partir de là en inventer de nouvelles), il faut nécessairement se souvenir des œuvres elles-mêmes (et les analyser, y réfléchir, chercher à les comprendre). C'est là, d'une certaine manière, une des singularités du roman que d'exiger, pour qu'on puisse le définir, la constante consultation de ses incarnations. On retrouve ici ce que Thomas Pavel appelle, dans La pensée du roman, la nature «coutumière » du roman, qui transmet ses techniques et ses pratiques non sous forme de règles ou de théories, mais sous celle de jurisprudences:

À défaut d'un code hérité, le roman a su profiter au cours de son évolution de la richesse de formes narratives disponibles. Aussi, avant le $\mathrm{XVIII}^{\mathrm{e}}$ siècle, la force du roman a-t-elle résidé moins dans un ensemble de règles explicites que dans la vigueur et la diversité des pratiques suivies. [...] Les règles de ce[s] pratique[s], le plus souvent incorporées silencieusement dans les œuvres ellesmêmes, ont pris, dans quelques rares occasions, non la forme du traité ou du discours dogmatique, mais celle des préfaces et des commentaires qui accompagnaient les nouvelles productions, un peu à la manière de la pensée légale dans les pays de droit coutumier ${ }^{15}$.

À l'époque classique où, comme l'écrit Judith Schlanger, «les livres n'appart[enaient] pas vraiment au passé historique, puisque la bibliothèque fai[sait] d'eux des contemporains éclatants» (DTE, p. 36), ce relais allait de soi et s'effectuait sans effort particulier. Mais, dans un

14. Henry James, «La leçon de Balzac» [1905], Du roman considéré comme un des beaux-arts, trad. de l'anglais par Chantal de Biasi, Paris, Christian Bourgois éditeur, 1987, p. 218-255.

15. Thomas Pavel, La pensée du roman, Paris, Gallimard, coll. «NRF Essais», 2003, p. 18. 
monde soumis à l'oubli, il est sans doute normal qu'il s'exprime non plus «silencieusement» ou dans quelques rares textes contingents, mais de façon explicite et comme une pratique soutenue.

Il me semble toutefois que cette spécificité du roman, c'està-dire la nécessité qu'il induirait, pour qu'on puisse en parler, de donner des exemples, de rappeler tel ou tel cas précis plutôt que tel ou tel «modèle» général, ne suffit pas à tout expliquer. Si, au début du $\mathrm{xx}^{\mathrm{e}}$ siècle, les romanciers éprouvent le besoin de penser l'histoire de leur art, s'ils cherchent à ne pas perdre le lien avec le passé, c'est aussi parce que surgit à ce moment-là un rapport nouveau aux formes héritées. Le $\mathrm{xx}^{\mathrm{e}}$ siècle s'ouvre en demandant aux œuvres, pour reprendre l'expression de Jacques Rivière, de se «débarbouill [er] du passé ${ }^{16}$ », c'est-à-dire sinon d'oublier le passé, du moins d'être libres face à lui. Ce n'était pas la première fois, dans l'histoire de la littérature, que s'exprimait un désir de renouveau: qu'on songe à la Querelle des Anciens et des Modernes ou au désir des écrivains romantiques d'écrire pour leur temps propre. Ces demandes de rénovation suivaient cependant de longues périodes de continuité et de tradition, perçues comme des formes d'immobilisme ou de stagnation, et dont les cuvres apparaissaient assez solides, assez durables pour ne pas être oubliées au passage. On ne peut en dire autant pour le XIX ${ }^{\mathrm{e}}$ siècle, qu'il est difficile (et qu'il était difficile, pour les écrivains et les artistes du $\mathrm{xx}^{\mathrm{e}}$ siècle, même s'ils proclamaient le contraire) de considérer comme une époque de répétition ou de blocage, non plus que comme une époque de stabilité. Au contraire, jamais n'avait-on jusque-là vu autant de courants et de mouvements se succéder, ni, surtout, autant encouragé ni facilité le nouveau. C'est pourquoi la demande faite à la littérature, au début du $\mathrm{xx}^{\mathrm{e}}$ siècle, de rompre avec les formes du passé ne peut se comparer aux appels à la modernité formulés par Perrault, Stendhal ou Hugo en leur temps, pour qui il s'agissait non pas d'oublier les œuvres du passé mais de les augmenter de celles du présent. La demande du $\mathrm{xx}^{\mathrm{e}}$ siècle, qui se fait sur fond d'encombrement de la mémoire et de multiplication des formes nouvelles, serait, peut-on proposer, inverse: elle n'émanerait pas tant d'un besoin d'élargissement des possibilités de la littérature que, au contraire, d'un désir de repos ou d'apaisement, de simplification ou de décharge. Plaider

16. Jacques Rivière, Le roman d'aventure, Paris, Édition des Syrtes, 2000, p. 26. 
pour une littérature et des arts du présent et de l'avenir, comme l'ont fait tout au long du $\mathrm{xx}^{\mathrm{e}}$ siècle les avant-gardes (pensons aux surréalistes, qui veulent «en finir avec l'ancien régime de l'esprit ${ }^{17}$ », aux futuristes, qui disent se trouver "sur le promontoire extrême des siècles! À quoi bon regarder derrière nous, du moment qu'il nous faut défoncer les vantaux de l'impossible? Le Temps et l'espace sont morts hier ${ }^{18}$ », ou encore aux écrivains du Nouveau roman, pour qui la vie, «dans son mouvement incessant qui la fait se déplacer toujours vers cette ligne mobile où parvient à un moment la recherche et où porte tout le poids de l'effort ${ }^{19}$ ", fait d'elle-même éclater les vieux cadres esthétiques), était un moyen en quelque sorte "positif» d'alléger l'héritage, de transformer en conquête ce qui, sur le plan de la mémoire et de la connaissance, était une perte ou une forme d'abandon, aussi nécessaires et féconds pouvaient-ils être. Autrement dit, face à la situation décrite par Nietzsche dans sa Seconde considération intempestive que le propre de l'homme moderne est de porter le poids toujours plus lourd des œuvres toujours plus nombreuses qui le précèdent, se délester de ce poids, pour qui veut créer, peut être vu comme une forme de libération ${ }^{20}$.

Mais cet allègement réclamé par les avant-gardes — ou cet « isolement», pour reprendre le terme de Valéry à propos de la poésie est problématique pour le roman. Le temps sans passé ou en rupture de passé des avant-gardes est pour la forme romanesque un temps étroit. D'abord pour des raisons de dimensions, la longueur propre au roman ayant partie liée à son inscription dans un temps dont on éprouve qu'il s'écoule et nous emporte avec lui. Ensuite aussi pour des raisons de propos et de matière, le roman racontant sinon l'expérience de la durée, tout au moins des expériences dont le propre est de durer. Dans un entretien publié dans Le Temps en novembre 1913, Proust lie spécifiquement le genre romanesque à la «substance»du temps long:

17. André Breton, Alentours III, dans Euvres complètes, $t$. I, Paris, Gallimard, coll. «Bibliothèque de la Pléiade», 1988, p. 904.

18. Filippo Tommaso Marinetti, «Manifeste du futurisme», Le Figaro, 20 février 1909, p. 1.

19. Nathalie Sarraute, L'Ère du soupçon, Paris, Gallimard, coll. «Idées», 1972 [1956], p. 77-78.

20. Friedrich Nietzsche, Seconde considération intempestive, ouvr. cité, chapitre IV, p. 102-112. 
De jeunes écrivains, avec qui je suis d'ailleurs en sympathie, préconisent $[\ldots]$ une action brève avec peu de personnages. Ce n'est pas ma conception du roman. Comment vous dire cela? Vous savez qu'il y a une géométrie plane et une géométrie dans l'espace. Eh bien, pour moi, le roman ce n'est pas seulement de la psychologie plane, mais de la psychologie dans le temps. Cette substance invisible du temps, j'ai tâché de l'isoler, mais pour cela il fallait que l'expérience pût durer. [...] les divers aspects qu'un même personnage aura pris aux yeux d'un autre, au point qu'il aura été comme des personnages successifs et différents, donneront - mais par cela seulement — la sensation du temps écoulée ${ }^{21}$.

On trouve une réflexion similaire chez Roger Martin du Gard, pourtant très éloigné de Proust sur le plan esthétique, dans une lettre adressée à Pierre Maragritis et recopiée dans son Journal en novembre 1915:

Sens-tu combien l'air impur des journaux, des partis politiques, des théories sociales, des discussions religieuses, des ergotages philosophiques, est pernicieux, est empoisonné pour la floraison de l'œuvre d'art? Je ne me trompe pas. Je n'exagère pas. Je sais bien tout ce qu'on peut dire. Être de son temps, communier avec les ardeurs et les spasmes contemporains, alimenter son inspiration de ce qu'il y a de plus capiteux dans la vie des foules fraternelles. [...] Je me retourne en arrière, je relis Barois. Où est l'œuvre d'art? Où est ce petit quelque chose qui pourrait survivre à l'époque, être de tous les temps, émouvoir le cour de tous les hommes? Est-ce l'affaire Dreyfus? Sont-ce les discussions documentées sur la foi, sur les dogmes, sur l'avenir de la croyance? Non. Ça c'est nourriture pour les contemporains, pour les amateurs d'actualités, ou bien, plus tard, pour quelques historiens curieux de choses mortes, périmées. Non. Si j'ai pu, par-ci parlà, atteindre par bribes à l'œuvre d'art, à l'œuvre éternelle, c'est dans tel ou tel mot sorti du cœur humain, vrai pour toutes les époques ${ }^{22}$.

Alors que tout s'accélère, que les avant-gardes se multiplient et que la poésie s'emploie à toucher à son «essence», invente l'écriture simultanée et automatique, le roman, dans un mouvement contraire

21. Cité par Élie-Joseph Blois, «Swann expliqué par Proust», Le Temps [13 novembre 1913], dans Essais et articles, Paris, Gallimard, coll. «Folio», 1994, p. 253.

22. Roger Martin du Gard, lettre à Pierre Maragritis [3 septembre 1915], Journal, éd. Claude Sicard, Paris, Gallimard, 1993, t. I, p. 654-655. 
et presque anachronique, cherche les moyens de dire la sensation du temps écoulé et du temps long, de distinguer ce qui peut survivre à l'époque, c'est-à-dire rester pour mémoire. La critique des romanciers, à la fois comme événement récent de l'histoire du roman et dans sa propension non pas à théoriser de façon abstraite mais à parler concrètement des œuvres d'autrui, serait, peut-on avancer comme hypothèse, lié à ce désir — ou à cette nécessité - d'aller contre l'allègement du temps et de la mémoire voulu ou accepté par les autres arts, de s'inscrire dans une durée plus large que le présent, d'admettre et d'accueillir sinon toutes les dettes - ce qui serait bien sûr impossible — du moins l'idée de dette, comme celle d'admiration. Il est d'ailleurs frappant, à cet égard, que les deux romanciers généralement retenus par l'histoire de la littérature comme les plus influents de la première moitié du $\mathrm{XIX}^{\mathrm{e}}$ siècle, à savoir Proust et Joyce, ont fortement inscrit au sein de leur œuvre des références d'ordre mémoriel (notamment Balzac et Mme de Sévigné pour le premier; Homère pour le second). Il y aurait en ce sens invitation à établir un rapport entre le temps long du roman et le temps également long auquel pense et travaille la critique des romanciers, et qu'on pourrait définir par le terme de reconnaissance. Que ce soit par l'établissement de filiations, le rappel d'influences ou son sens de la «coutume», la critique des romanciers cherche à ne pas oublier, à ne pas rompre, à ne pas perdre de vue ce qui la lie à une histoire. Même les romanciers qui se réclament de l'avant-garde se savent redevables du temps long et c'est tout sauf un hasard si les auteurs du Nouveau roman ont à ce point cherché à inscrire leur œuvre au sein d'une filiation ${ }^{23}$, non plus si, encore aujourd'hui, on entend des romanciers évoquer le roman balzacien ou le roman zolien comme des modèles périmés: ce sont des modèles périmés depuis bientôt un siècle, mais les romanciers, même lorsqu'ils se donnent l'air de chasser la mémoire, ont besoin de celle-ci, d'un rappel de ce que le roman a créé et de ce qui leur a été légué.

Judith Schlanger termine son article en s'interrogeant sur les raisons qui font que nous aimons lire ce que les écrivains disent de la littérature et de son histoire. Ce ne sont pas, propose-t-elle, des connaissances que nous allons chercher dans leurs essais, leurs

23. Voir entre autres, à ce sujet, l'article très éclairant de Katerine Gosselin, «Le Nouveau roman devant le roman ", dans Isabelle Daunais (dir.), Le roman vu par les romanciers, Québec, Nota bene, 2008, p. 103-128. 
commentaires, leurs entretiens ou leurs confessions (nous n'attendons d'eux «ni des manuels [...] ni l'information historique de pointe qui naît de travaux spécialisés et de recherches d'archives»; DTE, p. 38), mais une façon de comprendre comment on peut lier l'art et la vie: "Ces écrits nous apportent une prose méditative née dans la connivence privilégiée du commerce intime des lettres et de l'expérience personnelle» (DTE, p. 38). Plus précisément, c'est en ce qu'elle nous rapprocherait de l'expérience classique des lettres, définie comme une fréquentation «directe» des œuvres, peu importe où elles se situent dans l'échelle du temps, que nous aimerions lire la critique des écrivains et que nous la distinguerions de la critique savante. À cette explication très juste on peut en ajouter une autre: cette critique trouverait son intérêt, et le trouverait particulièrement s'agissant du roman, de ce qu'elle constitue une forme de mémoire. Dans un siècle (le $\mathrm{xxI}^{\mathrm{e}}$ comme le $\mathrm{xx}^{\mathrm{e}}$ ) qui a misé, dans presque tous les domaines, sur les ruptures, l'innovation et le renouveau, la mémoire du roman et le sens de l'histoire des romanciers formeraient un bien singulier, que nous reconnaîtrions comme tel. 